\title{
Gaussian Schell-model beams propagating through polarization gratings
}

\author{
Gemma Piquero \\ Departamento de Óptica, Universidad Complutense de Madrid, Ciudad Universitaria s/n, E-28040 Madrid, Spain \\ Riccardo Borghi \\ Dipartimento di Ingegneria Elettronica, Università Roma Tre and Istituto Nazionale per la Fisica della Materia, \\ Via della Vasca Navale 84, I-00146 Roma, Italy
}

Massimo Santarsiero

Dipartimento di Fisica, Università Roma Tre and Istituto Nazionale per la Fisica della Materia, Via della Vasca Navale 84, I-00146 Roma, Italy

Received August 4, 2000; revised manuscript received November 28, 2000; accepted December 4, 2000

The effects of polarization gratings on partially coherent beams are investigated by studying a Gaussian Schell-model beam impinging on a linear polarizer whose transmission axis varies periodically along one transverse direction. Analytical expressions for the beam polarization-coherence matrix after the grating are obtained. In particular, the evolution of the degree of polarization upon propagation is analyzed. Different behaviors of the output beam, depending on the beam parameters and on the period of the grating, are exhibited. In particular, it is shown that, by suitably choosing the latter quantities, it is possible to obtain not only any desirable value of the degree of polarization of the output beam but also particular distributions of such parameters across the transverse sections of the beam. (C) 2001 Optical Society of America

OCIS codes: $260.5430,030.0030,230.6080,050.2770$.

\section{INTRODUCTION}

Polarization gratings (PG's) are anisotropic optical elements of increasing interest for many applications, for example, measurement of the Stokes parameters by means of PG's, ${ }^{1,2}$ obtaining circularly polarized beams from unpolarized sources, ${ }^{3}$ and achieving duplicators and triplicators with $100 \%$ of efficiency. ${ }^{4}$ A further possible application that has recently been proposed ${ }^{5}$ is the process of synthesizing beams with peculiar characteristics of polarization in their transverse sections. ${ }^{6-10} \mathrm{Up}$ to now, the effects of PG's on optical beams have been studied on totally coherent beams. The aim of this paper is to extend such previous analyses to partially coherent incident beams. In particular, we use a Gaussian Schell-model (GSM) source, for which both the optical intensity and the degree of coherence have Gaussian profile. ${ }^{11}$ This kind of source has been proved to be useful in modeling real partially coherent and laser sources (see, for example, Ref. 11 and references therein). Applications of the GSM model to the case of partially polarized light beams have also been studied. ${ }^{12,13}$ In this paper we study a GSM beam impinging on a linear polarizer whose transmission axis varies periodically along a transverse coordinate. Analytical expressions of the beam coherence-polarization (BCP) matrix ${ }^{14,15}$ after the PG and upon free propagation are given. Polarization features, and in particular the evolution of the local degree of polarization $P$ upon propagation, are investigated as functions of the parameters of the input beam and of the period of the grating. It is shown that, for some values of the degree of coherence and small values of the propagation distance, the degree of polarization presents maxima and minima. Furthermore, by suitably choosing the characteristics of the PG, it is possible to obtain not only any value of $P$ of the propagated beam but even different distributions of such parameter in a transverse plane, both in the near and in the far zone.

The paper is structured as follows: In Section 2 we introduce the formalism and definitions to be used. We derive the analytical expressions of the elements of the $\mathrm{BCP}$ matrix just behind the polarization grating (Section 3) and upon propagation (Section 4). The results concerning the totally coherent, incoherent, and partially coherent cases are presented in Sections 5, 6, and 7, respectively, which also include the main general conclusions for each case. Finally, in Section 8 brief concluding remarks are made.

\section{FORMALISM AND DEFINITIONS}

For the sake of simplicity, we shall limit ourselves to the two-dimensional case. Let us recall that, for a quasimonochromatic field propagating along the $z$ axis of a suitable reference frame $(x, z)$, the BCP matrix is defined as $^{14,15}$

$$
\hat{J}\left(x_{1}, x_{2}, z\right)=\left[\begin{array}{ll}
J_{x x}\left(x_{1}, x_{2}, z\right) & J_{x y}\left(x_{1}, x_{2}, z\right) \\
J_{y x}\left(x_{1}, x_{2}, z\right) & J_{y y}\left(x_{1}, x_{2}, z\right)
\end{array}\right],
$$


where

$$
\begin{aligned}
J_{p q}\left(x_{1}, x_{2}, z\right) & \\
\quad & \left\langle E_{p}^{*}\left(x_{1}, z ; t\right) E_{q}\left(x_{2}, z ; t\right)\right\rangle \quad(p, q=x, y) .
\end{aligned}
$$

The angle brackets denote time average and $E_{p}(p$ $=x, y)$ is a Cartesian component of the time-dependent electric field. In addition, the following relationships among the elements of the BCP matrix hold:

$$
\begin{array}{r}
J_{x y}\left(x_{1}, x_{2}, z\right)=J_{y x}^{*}\left(x_{2}, x_{1}, z\right), \\
\left|J_{p q}\left(x_{1}, x_{2}, z\right)\right|^{2} \leqslant J_{p p}\left(x_{1}, x_{1}, z\right) J_{q q}\left(x_{2}, x_{2}, z\right) \\
(p, q=x, y) .
\end{array}
$$

The degree of polarization can be evaluated at a typical point $(x, z)$ starting from the elements of the BCP matrix evaluated for the points $x_{1}=x_{2}=x$ and is given by ${ }^{11}$

$$
\begin{aligned}
& P(x, z) \\
& \quad=\left\{\frac{\left[J_{x x}(x, x, z)-J_{y y}(x, x, z)\right]^{2}+4\left|J_{x y}(x, x, z)\right|^{2}}{\left[J_{x x}(x, x, z)+J_{y y}(x, x, z)\right]^{2}}\right\}^{1 / 2} .
\end{aligned}
$$

In addition, we can define an equivalent mutual intensity of the beam as ${ }^{14}$

$$
J_{\mathrm{eq}}\left(x_{1}, x_{2}, z\right)=J_{x x}\left(x_{1}, x_{2}, z\right)+J_{y y}\left(x_{1}, x_{2}, z\right) .
$$

In particular, the optical intensity, say, $I(x, z)$, is given by

$$
I(x, z)=J_{\mathrm{eq}}(x, x, z) .
$$

In fact, if no anisotropic elements are used, all propagation, diffraction, and interference phenomena will be correctly described by replacing the beam written in terms of the BCP matrix by the beam describable in scalar terms, when its mutual intensity is given by Eq. (6). ${ }^{16}$

It should be noted that application of the BCP formalism to radiated fields that are not describable by the beam model requires some caution. This approximation applies when the $z$ component of the field can be neglected and the light is quasi-monochromatic. The need for a complete vectorial treatment for certain cases, such as blackbody radiation, ${ }^{17}$ has been demonstrated. ${ }^{11,18}$

\section{GAUSSIAN SCHELL-MODEL BEAMS AFTER THE POLARIZATION GRATING}

Let us now consider, for simplicity, an unpolarized GSM source at the plane $z=0$, whose $\mathrm{BCP}$ matrix is of the form

$$
\hat{J}^{s}\left(x_{1}, x_{2}\right)=J_{\mathrm{sc}}\left(x_{1}, x_{2}, 0\right)\left[\begin{array}{ll}
1 & 0 \\
0 & 1
\end{array}\right]
$$

where

$$
J_{\mathrm{sc}}\left(x_{1}, x_{2}, 0\right)=I_{0} \exp \left(-\frac{x_{1}^{2}+x_{2}^{2}}{4 \sigma_{I_{0}}^{2}}\right) \exp \left[-\frac{\left(x_{1}-x_{2}\right)^{2}}{2 \sigma_{\mu_{0}}^{2}}\right],
$$

is the usual mutual intensity of a GSM source in the scalar case. Here $I_{0}$ is a constant intensity factor and $\sigma_{I_{0}}^{2}$ and $\sigma_{\mu_{0}}^{2}$ are the variances of the transverse intensity profile and of the degree of coherence of the source, respectively. The subscript sc denotes that $J_{\mathrm{sc}}$ coincides with the mutual intensity of a scalar GSM source.

Now we will study the case of a PG placed at the source plane. The PG that we are going to consider is a linear polarizer in which the angle between the transmission axes and $x$ axis is a linear function of $x$. The BCP matrix of the beam at the output of the $\mathrm{PG}$ is evaluated as follows, ${ }^{15}$

$$
\begin{aligned}
\hat{J}\left(x_{1}, x_{2}, 0\right)= & J_{\mathrm{sc}}\left(x_{1}, x_{2}, 0\right)\left[\begin{array}{cc}
C_{1}^{2} & C_{1} S_{1} \\
C_{1} S_{1} & S_{1}^{2}
\end{array}\right] \\
& \times\left[\begin{array}{ll}
1 & 0 \\
0 & 1
\end{array}\right]\left[\begin{array}{cc}
C_{2}^{2} & C_{2} S_{2} \\
C_{2} S_{2} & S_{2}^{2}
\end{array}\right],
\end{aligned}
$$

where

$$
C_{j}=\cos \left(\gamma x_{j}\right), \quad S_{j}=\sin \left(\gamma x_{j}\right) \quad(j=1,2),
$$

with $\gamma=\pi / L, L$ being the period of the grating. With use of Eqs. (10) and (11), the elements of $\hat{J}\left(x_{1}, x_{2}, 0\right)$ turn out to be

$$
\begin{aligned}
J_{x x}\left(x_{1}, x_{2}, 0\right)= & \frac{1}{2} J_{\mathrm{sc}}\left(x_{1}, x_{2}, 0\right) \cos \left[\gamma\left(x_{1}-x_{2}\right)\right] \\
& \times\left\{\cos \left[\gamma\left(x_{1}-x_{2}\right)\right]\right. \\
& \left.+\cos \left[\gamma\left(x_{1}+x_{2}\right)\right]\right\}, \\
J_{y y}\left(x_{1}, x_{2}, 0\right)= & \frac{1}{2} J_{\mathrm{sc}}\left(x_{1}, x_{2}, 0\right) \cos \left[\gamma\left(x_{1}-x_{2}\right)\right] \\
& \times\left\{\cos \left[\gamma\left(x_{1}-x_{2}\right)\right]\right. \\
& \left.-\cos \left[\gamma\left(x_{1}+x_{2}\right)\right]\right\}, \\
J_{x y}\left(x_{1}, x_{2}, 0\right)= & \frac{1}{2} J_{\mathrm{sc}}\left(x_{1}, x_{2}, 0\right) \cos \left[\gamma\left(x_{1}-x_{2}\right)\right] \\
& \times\left\{\sin \left[\gamma\left(x_{1}+x_{2}\right)\right]\right. \\
& \left.-\sin \left[\gamma\left(x_{1}-x_{2}\right)\right]\right\} .
\end{aligned}
$$

They represent a partially coherent source with a nonuniform state of polarization. The field is linearly polarized at any point on the source plane, but the polarization azimuth depends on the transverse coordinate. Accordingly, the degree of polarization of the emerging field is unitary everywhere across the transverse section. This can be easily verified by using Eq. (5) with Eqs. (12)-(14). In Section 4 we will study the propagation features of the beam radiated by such a source.

\section{FREE PROPAGATION AFTER THE POLARIZATION GRATING}

We will use the Fresnel propagation formula ${ }^{19}$ to evaluate the BCP elements of the beam described by Eqs. (12)-(14) at any plane $z=$ constant. Then, for each element $J_{p q}$ of the $\mathrm{BCP}$ matrix, we have 
$J_{p q}\left(x_{1}, x_{2}, z\right)$

$$
\begin{aligned}
= & \iint J_{p q}\left(\xi_{1}, \xi_{2}, 0\right) K^{*}\left(x_{1}, \xi_{1}, z\right) \\
& \times K\left(x_{2}, \xi_{2}, z\right) \mathrm{d} \xi_{1} \mathrm{~d} \xi_{2} \quad(p, q=x, y),
\end{aligned}
$$

where the propagation kernel is ${ }^{19}$

$$
K(x, \xi, z)=\sqrt{\frac{-i}{\lambda z}} \exp \left(i \frac{2 \pi z}{\lambda}\right) \exp \left[\frac{i \pi}{\lambda z}(x-\xi)^{2}\right],
$$

$\lambda$ being the wavelength.

From Eqs. (12)-(16) and after lengthy but straightforward calculations, we obtain the following BCP matrix for the propagated beam:

$$
\hat{\mathcal{J}}(s, t, z)=\mathcal{J}_{\mathrm{sc}}(s, t, z)\left[\begin{array}{ll}
h_{x x}(s, t, z) & h_{x y}(s, t, z) \\
h_{x y}^{*}(s, t, z) & h_{y y}(s, t, z)
\end{array}\right],
$$

with $s=\left(x_{1}+x_{2}\right) / 2, t=x_{1}-x_{2}$ and

$$
\mathcal{J}_{\mathrm{sc}}(s, t, z)=J_{\mathrm{sc}}\left(x_{1}, x_{2}, z\right),
$$

where

$$
\begin{aligned}
& \mathcal{J}_{\mathrm{sc}}(s, t, z) \\
& \quad=I_{0} \frac{\exp \left(-i \delta_{z} s t\right)}{F_{z}} \exp \left(i \frac{\delta_{z} s t}{F_{z}^{2}}\right) \exp \left[-\frac{\left(\alpha s^{2}+\beta t^{2}\right)}{F_{z}^{2}}\right]
\end{aligned}
$$

is the mutual intensity of the GSM beam, ${ }^{20}$ which has been written in the form of Eq. (19) for simplicity in the calculations. Here

$$
\begin{aligned}
F_{z}^{2} & =1+\frac{(\lambda z / \pi)^{2}}{4 \sigma_{I_{0}}^{2}}\left(\frac{1}{4 \sigma_{I_{0}}^{2}}+\frac{1}{\sigma_{\mu_{0}}^{2}}\right), \\
\alpha & =\frac{1}{2 \sigma_{I_{0}}^{2}}, \\
\beta & =\frac{1}{8 \sigma_{I_{0}}^{2}}+\frac{1}{2 \sigma_{\mu_{0}}^{2}}, \\
\delta_{z} & =\frac{2 \pi}{\lambda z} .
\end{aligned}
$$

The elements inside the matrix in Eq. (17) are

$$
\begin{aligned}
& h_{x x}(s, t, z)=\frac{1}{4}+\frac{1}{8}\left\{\exp \left(-\frac{4 \alpha \gamma^{2}}{\delta_{z}^{2} F_{z}^{2}}\right) G_{+}\left(\frac{4 \alpha \gamma s}{\delta_{z} F_{z}^{2}}, \frac{2 \gamma t}{F_{z}^{2}}\right)\right. \\
& +\exp \left[-\frac{(\alpha+4 \beta) \gamma^{2}}{\delta_{z}^{2} F_{z}^{2}}\right] \\
& \times\left[\exp \left(\frac{2 i \delta_{z} \gamma s-4 \beta \gamma t}{\delta_{z} F_{z}^{2}}\right)\right. \\
& \times G_{+}\left[\frac{2 \alpha \gamma s}{\delta_{z} F_{z}^{2}}, \frac{\left(\delta_{z} t+2 \gamma\right) \gamma}{\delta_{z} F_{z}^{2}}\right] \\
& +\exp \left(\frac{-2 i \gamma \delta_{z} s+4 \beta \gamma t}{\delta_{z} F_{z}^{2}}\right) \\
& \left.\left.\times G_{+}\left[\frac{2 \alpha \gamma s}{\delta_{z} F_{z}^{2}}, \frac{\left(\delta_{z} t-2 \gamma\right) \gamma}{\delta_{z} F_{z}^{2}}\right]\right]\right\}, \\
& h_{y y}(s, t, z)=\frac{1}{4}+\frac{1}{8}\left\{\exp \left(-4 \frac{\alpha \gamma^{2}}{\delta_{z}^{2} F_{z}^{2}}\right) G_{+}\left(\frac{4 \alpha \gamma s}{\delta_{z} F_{z}^{2}}, \frac{2 \gamma t}{F_{z}^{2}}\right)\right. \\
& -\exp \left[-\frac{(\alpha+4 \beta) \gamma^{2}}{\delta_{z}^{2} F_{z}^{2}}\right] \\
& \times\left[\exp \left(\frac{2 i \gamma \delta_{z} s-4 \beta \gamma t}{\delta_{z} F_{z}^{2}}\right)\right. \\
& \times G_{+}\left[\frac{2 \alpha \gamma s}{\delta_{z} F_{z}^{2}}, \frac{\left(\delta_{z} t+2 \gamma\right) \gamma}{\delta_{z} F_{z}^{2}}\right] \\
& +\exp \left(\frac{-2 i \gamma \delta_{z} s+4 \beta \gamma t}{\delta_{z} F_{z}^{2}}\right) \\
& \left.\left.\times G_{+}\left[\frac{2 \alpha \gamma s}{\delta_{z} F_{z}^{2}}, \frac{\left(\delta_{z} t-2 \gamma\right) \gamma}{\delta_{z} F_{z}^{2}}\right]\right]\right\}, \\
& h_{x y}(s, t, z)=\frac{1}{8 i}\left(\exp \left[-\frac{(\alpha+4 \beta) \gamma^{2}}{\delta_{z}^{2} F_{z}^{2}}\right]\right. \\
& \times\left\{\exp \left(\frac{2 i \gamma \delta_{z} s-4 \beta \gamma t}{\delta_{z} F_{z}^{2}}\right)\right. \\
& \times G_{+}\left[\frac{2 \alpha \gamma s}{\delta_{z} F_{z}^{2}}, \frac{\left(\delta_{z} t+2 \gamma\right) \gamma}{\delta_{z} F_{z}^{2}}\right] \\
& -\exp \left(\frac{-2 i \gamma \delta_{z} s+4 \beta \gamma t}{\delta_{z} F_{z}^{2}}\right) \\
& \left.\times G_{+}\left[\frac{2 \alpha \gamma s}{\delta_{z} F_{z}^{2}}, \frac{\left(\delta_{z} t-2 \gamma\right) \gamma}{\delta_{z} F_{z}^{2}}\right]\right\} \\
& \left.-\exp \left(-\frac{4 \alpha \gamma^{2}}{\delta_{z}^{2} F_{z}^{2}}\right) G_{-}\left(\frac{4 \alpha \gamma s}{\delta_{z} F_{z}^{2}}, \frac{2 \gamma t}{F_{z}^{2}}\right)\right)
\end{aligned}
$$

and

$$
G_{ \pm}(x, y)=\exp (-x) \exp (i y) \pm \exp (x) \exp (-i y) .
$$

Although elementary, Eqs. (24)-(26) cannot be read in a simple way. In order to obtain some more-immediate results, let us derive the expression of the equivalent mu- 
tual intensity. Starting from Eqs. (17)-(27) it is possible to obtain the expression of the equivalent mutual intensity $\mathcal{J}_{\text {eq }}(s, t, z)$, that is,

$$
\begin{aligned}
\mathcal{J}_{\text {eq }}(s, t, z)= & \mathcal{J}_{x x}(s, t, z)+\mathcal{J}_{y y}(s, t, z) \\
= & \mathcal{J}_{\mathrm{sc}}(s, t, z)\left[\frac{1}{2}+\frac{1}{4} \exp \left(-\frac{4 \alpha \gamma^{2}}{\delta_{z}^{2} F_{z}^{2}}\right)\right. \\
& \left.\times G_{+}\left(\frac{4 \alpha \gamma s}{\delta_{z} F_{z}^{2}}, \frac{2 \gamma t}{F_{z}^{2}}\right)\right]
\end{aligned}
$$

and of the optical intensity, that is,

$$
\begin{aligned}
I_{\mathrm{eq}}(x, z)= & \mathcal{J}_{\mathrm{eq}}(x, 0, z) \\
= & \frac{I_{0}}{2 F_{z}} \exp \left(\frac{-\alpha x^{2}}{F_{z}^{2}}\right)\left[1+\exp \left(\frac{-4 \alpha \gamma^{2}}{\delta_{z}^{2} F_{z}^{2}}\right)\right. \\
& \left.\times \cosh \left(\frac{4 \alpha \gamma x}{\delta_{z} F_{z}^{2}}\right)\right]
\end{aligned}
$$

Note that this expression can be expressed as the sum of three decentered Gaussians, corresponding to the three beams diffracted by the PG. Furthermore, from the definition in Eq. (6) and from Eqs. (17)-(26) the degree of polarization turns out to be

$P(x, z)$

$$
=\left\{\frac{\left[h_{x x}(x, 0, z)-h_{y y}(x, 0, z)\right]^{2}+4\left|h_{x y}(x, 0, z)\right|^{2}}{\left[h_{x x}(x, 0, z)+h_{y y}(x, 0, z)\right]^{2}}\right\}^{1 / 2} .
$$

Equation (30) gives the degree of polarization at any point in the half-space after the polarization grating.

A rather simple expression of the degree of polarization can be given in the far-field limit. In such a case it is convenient to introduce the angular variables $\theta_{s}=\left(x_{1}\right.$ $\left.+x_{2}\right) /(2 z)$ and $\theta_{t}=\left(x_{1}-x_{2}\right) / z$. Then the analytical expressions for the elements of the BCP matrix at the far field are given by

$$
\begin{aligned}
h_{x x}^{(\infty)}\left(\theta_{s}, \theta_{t}\right)= & \frac{1}{4}+\frac{1}{2} \exp \left[-\frac{(\alpha+4 \beta) \gamma^{2}}{4 \alpha \beta}\right] \\
& \times \cosh \left(\frac{\gamma \pi \theta_{s}}{\beta \lambda}\right) \cosh \left(\frac{2 \gamma \pi \theta_{t}}{\alpha \lambda}\right) \\
& +\frac{1}{4} \exp \left(-\frac{\gamma^{2}}{\beta}\right) \cosh \left(\frac{2 \gamma \pi \theta_{s}}{\beta \lambda}\right) \\
h_{y y}^{(\infty)}\left(\theta_{s}, \theta_{t}\right)= & \frac{1}{4}-\frac{1}{2} \exp \left[-\frac{(\alpha+4 \beta) \gamma^{2}}{4 \alpha \beta}\right] \\
& \times \cosh \left(\frac{\gamma \pi \theta_{s}}{\beta \lambda}\right) \cosh \left(\frac{2 \gamma \pi \theta_{t}}{\alpha \lambda}\right) \\
& +\frac{1}{4} \exp \left(-\frac{\gamma^{2}}{\beta}\right) \cosh \left(\frac{2 \gamma \pi \theta_{s}}{\beta \lambda}\right)
\end{aligned}
$$

$$
\begin{aligned}
h_{x y}^{(\infty)}\left(\theta_{s}, \theta_{t}\right)= & \frac{1}{4 i}\left\{\exp \left(-\frac{\gamma^{2}}{\beta}\right) \sinh \left(\frac{2 \gamma \pi \theta_{s}}{\beta \lambda}\right)\right. \\
& -2 \exp \left[-\frac{(\alpha+4 \beta) \gamma^{2}}{4 \alpha \beta}\right] \\
& \left.\times \sinh \left(\frac{2 \gamma \pi \theta_{t}}{\alpha \lambda}\right) \cosh \left(\frac{\gamma \pi \theta_{s}}{\beta \lambda}\right)\right\} .
\end{aligned}
$$

From the above equations the degree of polarization at the far field turns out to be

$P(\theta)$

$$
=\frac{\left\{4 \exp \left[\frac{\gamma^{2}(3 \alpha-4 \beta)}{2 \alpha \beta}\right] \cosh ^{2}\left(\frac{\gamma \pi \theta}{\beta \lambda}\right)+\sinh ^{2}\left(\frac{2 \gamma \pi \theta}{\beta \lambda}\right)\right\}^{1 / 2}}{\exp \left(\frac{\gamma^{2}}{\beta}\right)+\cosh \left(\frac{2 \gamma \pi \theta}{\beta \lambda}\right)} .
$$

In the following we will study the influence of the parameters of the GSM beam and the PG characteristics on the evolution of the degree of polarization with respect to $z$.

\section{FULLY COHERENT BEAM}

In this section we study the polarization characteristics of fully coherent Gaussian beams passing through a PG. Such a case corresponds to the set $\sigma_{\mu_{0}} \rightarrow \infty$ in the previous formulas. As was pointed out above, the degree of polarization is unitary at any point on the plane $z=0$. When the beam propagates along the $z$ axis, the degree of polarization $P$ changes depending on the period of the grating and on the beam width. Figure 1 shows the behavior of $P$ as a function of $z$ for $x=0$ and for different values of $L / \sigma_{I_{0}}$. It is clear from this figure that when $L \gg \sigma_{I_{0}}$ the degree of polarization is approximately equal to 1 for any value of $z$. This is not surprising, because in such limit the PG behaves as a simple linear polarizer

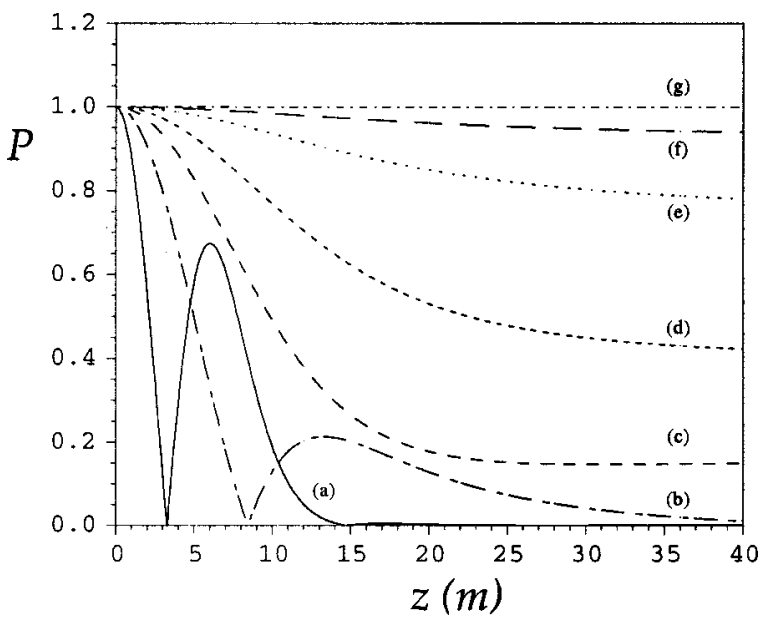

Fig. 1. Degree of polarization $P$ for an incident fully coherent beam on axis versus $z$ for different values of $L / \sigma_{I_{0}}$ : (a) 2 , (b) 3 , (c) 4, (d) 5, (e) 7, (f) 10, and (g) 30. The value of the wavelength is $\lambda=2 \pi \times 10^{-4} \mathrm{~mm}$. 


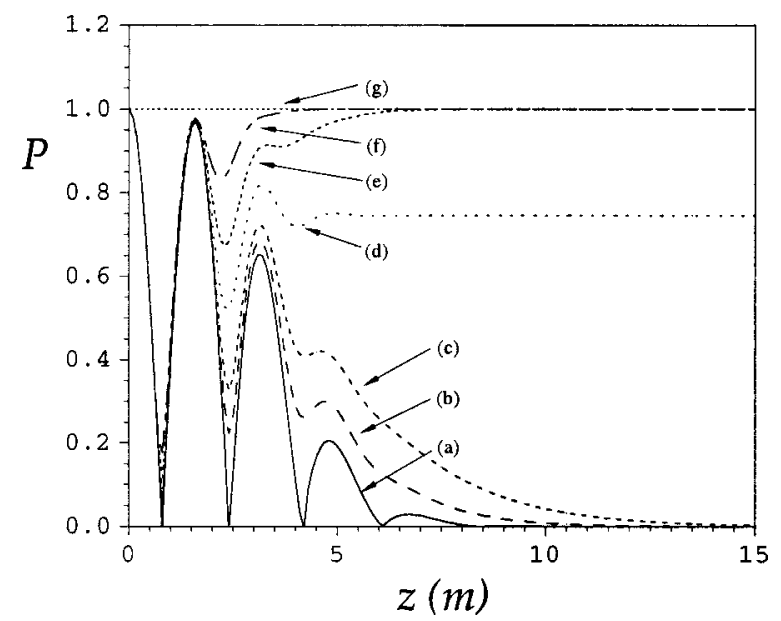

Fig. 2. Degree of polarization $P$ for an incident fully coherent beam versus $z$ for different values of $x / x_{L}$ : (a) 0 , (b) 0.2 , (c) 0.3 , (d) 0.5 , (e) 0.7 , (f) 1 , and (g) $3 . L / \sigma_{I_{0}}=1$ and $\lambda=2 \pi$ $\times 10^{-4} \mathrm{~mm}$.

and the emerging field is almost uniformly linearly polarized everywhere. On the other hand, by suitably choosing the value of $L / \sigma_{I_{0}}$ it is possible to obtain in the far field any desired value of $P$ at the center of the beam (see Fig. 1). For sufficiently small values of $L / \sigma_{I_{0}}$ it is possible to completely depolarize the beam on the axis. When $L$ further decreases, the $P$ parameter shows an increasingly complex behavior, presenting zeros for some values of $z$. Such behavior is evidenced in Fig. 2, where $P$ is shown for a small value of $L / \sigma_{I_{0}}$, presenting a seemingly strange behavior. To demonstrate this, in Fig. 2 we represent $P$ versus $z$ for $L / \sigma_{I_{0}}=1$ and for different values of $x / x_{L}$, with $x_{L}=\lambda z / L$. The degree of polarization for small values of $z$ shows maxima and minima. This behavior can be explained in a simple way as follows. In order to give an intuitive physical explanation, let us assume for simplicity that $\sigma_{I_{0}} \rightarrow \infty$. The latter can be thought of as the superposition of two mutually uncorrelated circularly polarized plane waves with opposite helicity (see Fig. 3). Let us consider separately the effect of the PG on each component wave. The first one gives rise to two circularly polarized plane waves corresponding to the zero and the first diffraction order, which propagate along different directions with opposite helicity. ${ }^{1}$ As a consequence, the superposition of the two orders produces a linearly, although nonuniformly, polarized transversal pattern, whose azimuth changes linearly with the transverse coordinate. Such pattern shifts transversally, as $z$ increases, along an angle fixed by the propagation directions of the diffraction orders. In particular, across the plane $z=0$, the polarization pattern reproduces the structure of the PG. Exactly the same occurs for the other component of the incident field, but the corresponding pattern shifts in the opposite direction. Thus, across certain planes, the polarization states of one pattern coincide with those of the other one at any point [see Fig. $4(a)$. In these cases the degree of polarization of the resulting field is unitary across the whole transverse plane. On the other hand, planes will exist where the polarization states of the two patterns are orthogonal to each other [see Fig. 4(b)], so that, since the component plane waves are mutually uncorrelated, the degree of polarization is expected to vanish at any point. It is easy to calculate that $L^{2} / \lambda$ is the distance between the planes with maximum (or minimum) values of $P$. This takes account of the behavior of the degree of polarization shown in Fig. 2. There, owing to the finite transverse extension of the incident Gaussian beam, the diffracted orders overlap only in a finite range of $z$ values. For higher values of $z$ the degree of polarization shows a more regular behavior. In particular, for small values of $x$ the field turns out to be unpolarized, whereas for larger values of $x$ the degree of polarization approaches 1 . This is explained by the fact that the zero order is completely unpolarized, whereas

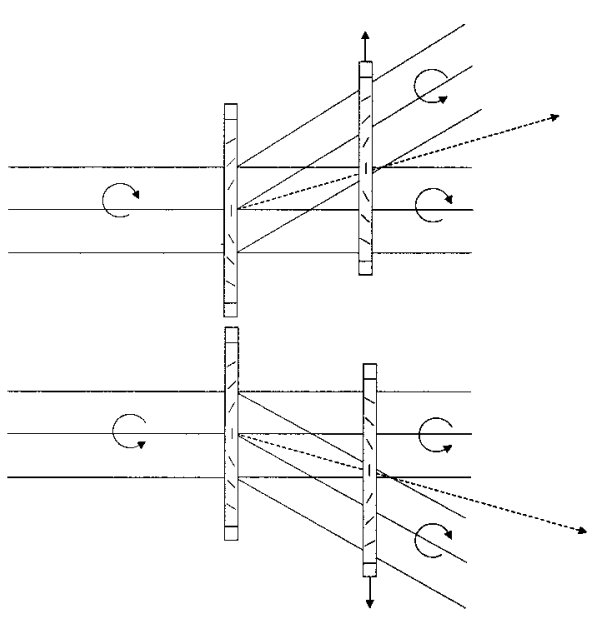

Fig. 3. Effect of the polarization grating on circularly polarized incident beams.

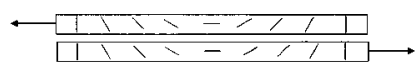

(a)

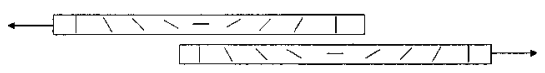

(b)

Fig. 4. Superposition scheme of two polarization patterns giving rise to a (a) maximum value and (b) a minimum value of $P$.

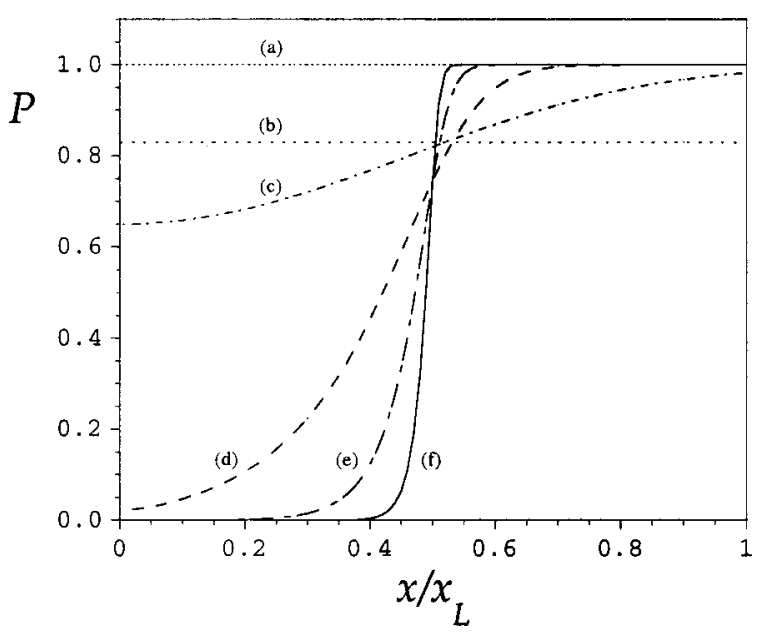

Fig. 5. Degree of polarization $P$ versus $x / x_{L}$ for different values of $z$ : (a) 0 , (b) $0.3 \mathrm{~m}$, (c) $3.2 \mathrm{~m}$, (d) $6.4 \mathrm{~m}$, (e) $12 \mathrm{~m}$, and (f) $30 \mathrm{~m}$. $L / \sigma_{I_{0}}=1$ and $\lambda=2 \pi \times 10^{-4} \mathrm{~mm}$. 
the \pm 1 orders are completely polarized ${ }^{1}$ and, at a suitable distance from the PG, the diffracted orders are spatially separated. This is demonstrated by Fig. 5, where the distribution of the degree of polarization is plotted across different transverse planes. In particular, the degree of polarization varies with the transverse coordinate $x$, and its behavior depends on the propagation distance $z$. This figure suggests the possibility of synthesizing a light beam endowed with unconventional local polarization features.

\section{INCOHERENT BEAM}

In the case of an incoherent beam, it is possible to obtain a simple expression for the BCP matrix. On placing $\sigma_{\mu_{0}}=0$ into Eqs. (17)-(26) we have

$$
\begin{aligned}
& \hat{\mathcal{J}}(s, t, z) \\
& \quad \times 2 \mathcal{J}_{\mathrm{sc}}^{\mathrm{inc}}(s, t, z) \\
& \quad\left[\begin{array}{cc}
1+\exp \left(-\frac{2 \pi^{2} \sigma_{I_{0}}^{2}}{L^{2}}\right) & 0 \\
0 & \left.1-\exp \left(-\frac{2 \pi^{2} \sigma_{I_{0}}^{2}}{L^{2}}\right)\right],
\end{array}\right.
\end{aligned}
$$

where $\mathcal{J}_{\mathrm{sc}}^{\text {inc }}(s, t, z)$ is the mutual intensity of the beam radiated by a planar incoherent Gaussian source. From Eq. (35) the state of polarization turns out to be the same everywhere in the space; i.e., the output beam is uniformly polarized. The degree of polarization $P$ can be evaluated as

$$
P=\exp \left(-\frac{2 \pi^{2} \sigma_{I_{0}}^{2}}{L^{2}}\right) .
$$

This value agrees with that of Eq. (34) evaluated for $\sigma_{\mu_{0}}$ $=0$, but Eq. (36) is valid for every point in the half-space $z>0$, not only in the far field. It should be noticed that $P$ depends only on the ratio $L / \sigma_{I_{0}}$. In particular, as for the coherent case, when $L \gg \sigma_{I_{0}}$ the state of polarization of the output beam is linear. In the opposite limit the BCP matrix tends to the unity matrix; i.e., the output beam is completely unpolarized. These results can be physically justified if the field emerging from the PG is thought of as the superposition of mutually uncorrelated cylindrical waves radiated by each element of the grating having polarization azimuths dictated by the structure of the PG and whose amplitude is fixed by the source size.

\section{PARTIALLY COHERENT BEAM}

The case of an incident partially coherent beam is much more complicated and varied than the cases treated above. Therefore we shall limit ourselves to giving results for some particular cases.

In Fig. 6, $P$ is represented versus $z$ for different values of the degree of coherence, with $x=0$ and $L / \sigma_{I_{0}}=1$. As the degree of coherence increases, the number of maxima and minima increases, too. In the central region, independent of the degree of coherence, the beam at the far

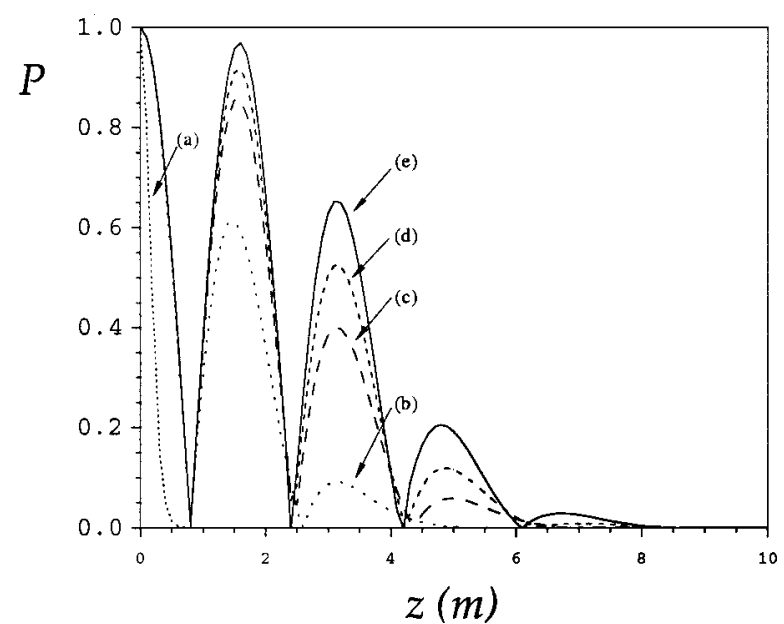

Fig. 6. Degree of polarization $P$ at $x / x_{L}=0$ versus $z$ for different values of the degree of coherence $\sigma_{\mu_{0}}$ : (a) $0.1 \mathrm{~mm}$, (b) $1 \mathrm{~mm}$, (c) $2 \mathrm{~mm}$, (d) $3 \mathrm{~mm}$, and (e) $\infty . L / \sigma_{I_{0}}=1$ and $\lambda=2 \pi$ $\times 10^{-4} \mathrm{~mm}$.

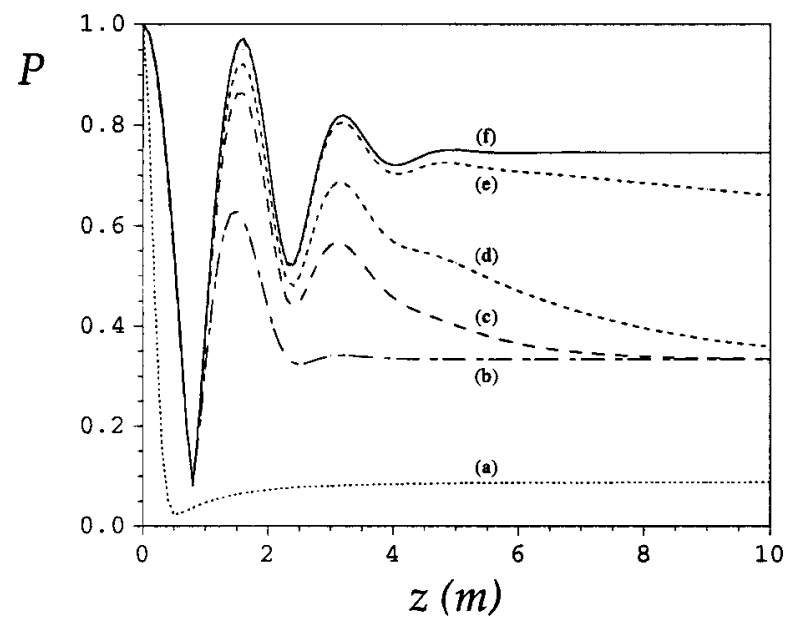

Fig. 7. Degree of polarization $P$ at $x / x_{L}=0.5$ versus $z$ for different values of $\sigma_{\mu_{0}}$ : (a) $0.1 \mathrm{~mm}$, (b) $1 \mathrm{~mm}$, (c) $2 \mathrm{~mm}$, (d) $3 \mathrm{~mm}$, (e) $10 \mathrm{~mm}$, and (f) $\infty . L / \sigma_{I_{0}}=1$ and $\lambda=2 \pi \times 10^{-4} \mathrm{~mm}$.

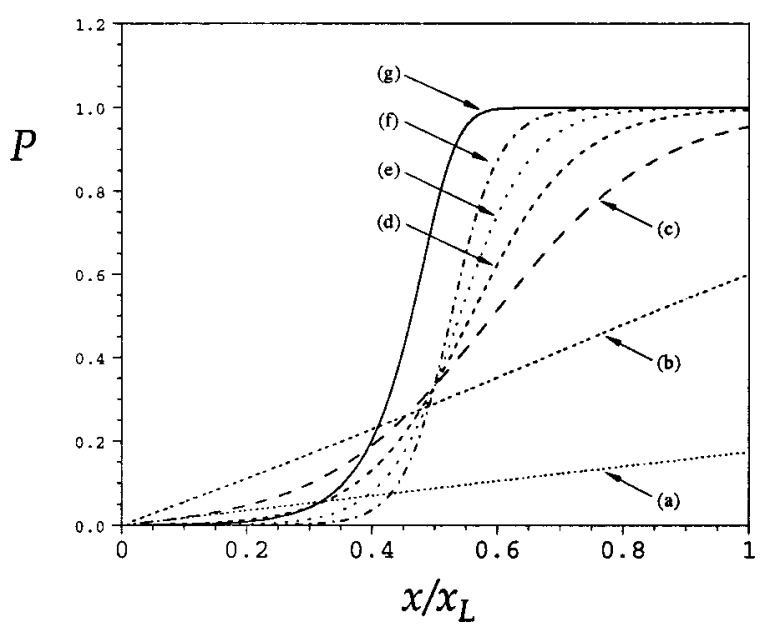

Fig. 8. Degree of polarization $P$ for a partially coherent incident beam versus $x / x_{L}$ at $z=10 \mathrm{~m}$ for different values of $\sigma_{\mu_{0}}$ : (a) 0.1 $\mathrm{mm}$, (b) $0.25 \mathrm{~mm}$, (c) $0.5 \mathrm{~mm}$, (d) $0.7 \mathrm{~mm}$, (e) $1 \mathrm{~mm}$, (f) $2 \mathrm{~mm}$, and (g) $\infty$. $L / \sigma_{I_{0}}=1$ and $\lambda=2 \pi \times 10^{-4} \mathrm{~mm}$. 
field is completely unpolarized. This behavior is not the same as for other points $x / x_{L}$, where the value of $P$ at the far field depends on $\sigma_{\mu_{0}}$ (see, for example, Fig. 7 for $x / x_{L}$ $=0.5$ ).

Furthermore, the uniformity of the degree of polarization at the transverse section of the beam changes with the degree of coherence, as well. In Fig. 8, $P$ is plotted versus $x / x_{L}$ for $z=10 \mathrm{~m}$. The distribution of values of $P$ changes from a constant value for the incoherent beam to the distribution shown by the solid curve, which corresponds to the fully coherent case.

\section{CONCLUSIONS}

The effects of PG's on partially coherent beams have been investigated by studying a Gaussian Schell-model beam impinging on a linear polarizer, whose transmission axis varies periodically along one transverse direction. The field emerging from the PG turns out to be partially coherent and with a nonuniform state of polarization. The propagated beam in the paraxial approximation is given in terms of its BCP matrix both in the near and in the far field. We have shown that the polarization characteristics of such a beam do not remain constant along $z$. In particular, the degree of polarization $P$ of such a beam upon propagation has been analyzed, and it has been shown that, by varying the characteristics of the GSM beam and of the grating, it is possible to modify the local degree of polarization, giving rise to a beam with a distribution of $P$ that changes not only from one point to another but also upon propagation.

\section{ACKNOWLEDGMENTS}

We thank Franco Gori for many helpful discussions during the preparation of this work. We acknowledge the project Azioni Integrate HI-1998-077 for support. One of the authors (G. Piquero) is grateful for support from the project PB97-295 and the program Becas Internacionales U.C.M/Flores Valles.

\section{REFERENCES}

1. F. Gori, "Measuring Stokes parameters by means of a polarization grating," Opt. Lett. 24, 584-586 (1999).
2. C. G. Someda, "Far field of polarization gratings," Opt. Lett. 24, 1657-1659 (1999).

3. P. Rochon, V. Drnoyan, and A. Natansohn, "Polarization holographic gratings in azopolymers for detecting and producing circularly polarized light," in 1998 International Conference on Applications of Photonic Technology III: Closing the Gap Between Theory, Developments, and Applications, G. A. Lampropoulos and R. A. Lessard, eds., Proc. SPIE 3491, 306-309 (2000).

4. J. Tervo and J. Turunen, "Paraxial-domain diffractive elements with $100 \%$ efficiency based on polarization gratings," Opt. Lett. 25, 785-786 (2000).

5. F. Gori, M. Santarsiero, R. Borghi, and G. Piquero, "Use of the van Cittert-Zernike theorem for partially polarized sources," Opt. Lett. 25, 1291-1293 (2000).

6. S. C. Tidwell, D. H. Ford, and W. D. Kimura, "Generating radially polarized beams interferometrically," Appl. Opt. 29, 2234-2239 (1990).

7. T. Erdogan, O. King, G. W. Wicks, D. G. Hall, E. Anderson, and M. J. Rooks, "Circularly symmetric operation of a concentric-circle-grating surface emitting AlGaAs/GaAs quantum well semiconductor laser," Appl. Phys. Lett. 60, 1921-1923 (1992).

8. P. L. Greene and D. G. Hall, "Properties and diffraction of vector Bessel-Gauss beams," J. Opt. Soc. Am. A 15, 30203027 (1998).

9. A. A. Tovar, "Production and propagation of cylindrically polarized Laguerre-Gaussian laser beams," J. Opt. Soc. Am. A 15, 2705-2711 (1998).

10. J. M. Movilla, G. Piquero, R. Martínez-Herrero, and P. M. Mejías, "Parametric characterization of non-uniformly polarized beams," Opt. Commun. 149, 230-234 (1998).

11. L. Mandel and E. Wolf, Optical Coherence and Quantum Optics (Cambridge U. Press, Cambridge, UK, 1995).

12. D. F. V. James, "Change of polarization of light beams on propagation in free space," J. Opt. Soc. Am. A 11, 16411643 (1994).

13. S. R. Seshadri, "Partially coherent Gaussian Schell-model electromagnetic beams," J. Opt. Soc. Am. A 16, 1373-1380 (1999).

14. F. Gori, "Matrix treatment for partially polarized, partially coherent beams," Opt. Lett. 23, 241-243 (1998).

15. F. Gori, M. Santarsiero, S. Vicalvi, R. Borghi, and G. Guattari, "Beam coherence-polarization matrix," J. Eur. Opt. Soc. A Pure Appl. Opt. 7, 941-951 (1998).

16. F. Gori, M. Santarsiero, R. Borghi, and G. Guattari, "The irradiance of partially polarized beams in a scalar treatment," Opt. Commun. 163, 159-163 (1999).

17. A. K. Jaiswal, G. P. Agrawal, and C. L. Mehta, "Coherence functions in the far field diffraction plane," Nuovo Cimento B 15, 295-307 (1973)

18. D. F. V. James, "Polarization of light radiated by black-body sources," Opt. Commun. 109, 209-214 (1994).

19. J. W. Goodman, Statistical Optics (Wiley, New York, 1985).

20. F. Gori, "Mode propagation of the field generated by Collett-Wolf sources," Opt. Commun. 46, 149-154 (1983). 